\title{
Optimal Bidding Strategies for Generation Companies in Electricity Markets with Transmission Capacity Constraints Taken into Account
}

\author{
Li Ma, Fushuan Wen, Yixin Ni, Senior Member, IEEE, and F. F. Wu, Fellow, IEEE
}

\begin{abstract}
In the electricity market environment, how to build optimal bidding strategies has become a major concern for generation companies. The deficiency of transmission capacity could lead to congestion, and as a result, the whole electricity market can then be actually divided into two or more submarkets. A direct consequence of transmission congestion is the change of competitive positions of generation companies concerned in the electricity market, and the optimal bidding strategies of them should accordingly be changed. In this paper, the problem of developing optimal bidding strategies for generation companies is systematically investigated with transmission capacity constraints taken into account. A stochastic optimization model is first formulated under the presumption that the bidding behaviors of rival generation companies could be modeled as normal probability distributions. An approach is next presented for solving the optimization problem using the well-known Monte Carlo simulation method and the genetic algorithm. Finally, a simple sample example and the modified IEEE 14-bus system are employed to illustrate the essential features of the proposed model and method.
\end{abstract}

Index Terms-- electricity market, Bidding strategy, congestion management, market power, Monte Carlo simulation, genetic algorithm.

\section{INTRODUCTION}

$\mathrm{n}$ the process of power industry restructuring, how to manage congestion caused by transmission capacity deficiency is a very important problem of extensive concern. Although transmission congestion also happened in the operation of the traditional monopoly power system, the problem could easily be solved by re-dispatching the outputs of generation units by the well-known preventive or corrective dispatching since changing the outputs of generation units does not lead to the reallocation o f economic benefits. However, in the electricity market environment, each generation company has the objective of maximizing its own benefit, and this is conflict with the objectives of other generation companies. Since generation re-dispatching can have an influence on the benefits of generation companies,

This work is jointly supported by a seeding fund project from the University of Hong Kong, and a specialized research fund for the doctoral program of higher education (SRFDP), China.

Li $\mathrm{Ma}$ is with Zhejiang University, Hangzhou, China(e-mail: mali_madi_mati(i)honail.com).

Fushuan Wen, Yixin Ni and F.F. Wu are with the University of Hong Kong. HK(e-mail: liwen@ee.hku.hk., yxni@ec.hku.hk, ffwu@ee.hku.hk). fairness is an important issue to be looked after in congestion management, in addition to the well-acknowledged power system security. Hence, the problem is much more complicated and difficult than that in the traditional power system. Moreover, the operation patterns of power systems in the electricity market environment may be very different from those in traditional power systems and, moreover, change more frequently since generation dispatching is bid-based rather than cost-based. In addition, since transmission systems were planned and constructed based on traditional generation planning and dispatching patterns, transmission congestion occurs more frequently in the new market environment than that in the traditional power system operation.

In the electricity market environment, transmission congestion management is not only a technical problem. Under some circumstances, transmission congestion could divide the whole electricity market into two or more submarkets and remarkably change the competitive positions of generation companies concerned in the electricity market. The profits of some generation companies will be reduced and those of others increased as a result of transmission congestion. It is hence expected that the prospected transmission congestion can have important impacts on the bidding strategies of generation companies.

To build optimal bidding strategies for generation companies or through this to investigate their potential abuse of market power in electricity markets is a very active research area and considerable amount of research work has been done $e^{[1-6]}$, and in Ref. [1] a comprehensive survey was made. Some research work has been done on examining the strategic behavior of some generation companies of unique geographical positions to intentionally cause transmission congestions so as to increase profits ${ }^{[7.8]}$, however, to the best of our knowledge, a systematic approach to develop optimal bidding strategies for generation companies with transmission congestion taken into account is still not available.

Many different methods are employed to manage transmission congestion in several operating electricity markets ${ }^{[9]}$. Obviously, different congestion management methods especially different settlement rules concerned could lead to different optimal bidding strategies for generation companies. Based on the congestion management method utilized in the former England and Wales electricity market in which the so-called uplift is used for congestion management, an optimal bidding strategy model is developed in this work. The framework of the developed method can be extended and 
applied to electricity markets with other congestion management methods employed, although, as expected, the details concerned need to be modified. The proposed model could be used to investigate the potential that generation companies manipulate the electricity market and exercise market power as a result of transmission capacity deficiency ${ }^{[10]}$. Based on the method proposed in Ref. [3] and assuming that the bidding behaviors of rival generation companies could be represented by normal probability distributions (an implicit assumption is actually made here that the electricity market studied is operating in a mature state and thus the rivals' bidding behaviors can be estimated as probability distributions based on historical bidding data), the problem of building optimal bidding strategies for generation companies is formulated as a stochastic optimization model and solved by the well-known Monte Carlo simulation method and Genetic Algorithm. Finally, a simple example and the modified IEEE 14-bus system are served for illustrating the basic feature of the proposed model and method.

\section{INTRODUCTION OF THE ELECTRICITY MARKET STUDIED}

The research work in this paper is based on the so-called single-buyer electricity market model. In this kind of electricity markets, generation companies (GENCOs) act as sellers and the transmission company or a power trading and dispatching center as the single buyer purchasing power energy from GENCOs based on load forecasting and bids from GENCOs and selling to consumers. The power trading and dispatching center is responsible for market trading and power system operation. The power trading is done daily and GENCOs are required to bid for the 48 (half-hour) periods in the next trading day before it starts. It is also assumed that GENCOs are required to bid a linear non-decreasing supply function and the uniform market clearing price (MCP) is employed for settlement.

When the unconstrained generation dispatching could result in transmission congestion, the uplift was used to manage congestion in the former England and Wales electricity market as stated before. There are two prices associated: power purchase price (PPP) paid to GENCOs by the single buyer and power sale price (PSP) sold to consumers (here consumers refer to distribution companies and large customers). $P P P=\lambda+C_{p}$, and $\lambda$ is the MCP and $C_{p}$ the capacity payment calculated by $C_{p}=\mathrm{LOLP} \times(\mathrm{VLL}-\lambda)$. LOLP is the Loss Of Load Probability and VLL the Value of Lost Load. In the former England and Wales electricity market, LOLP was generally around 0.001 and VLL 20003000 f/MWh. The energy prices paid to GENCOs can be classified into four kinds. When a generation unit is included in the unconstrained generation dispatching and also actually operated in the trading day, the price paid is PPP. When a generation unit is included in the unconstrained generation dispatching but doesn't be actually operated as required by the power trading and dispatching center due to transmission congestion, the price paid is PPP minus the unit's actual bid. When a generating unit bids but is not included in the unconstrained generation dispatching, however it is required to generate power by the power trading and dispatching center in the real-time operation, the price paid is the unit's actual bid plus $C_{p}$. When a generating unit bids but is not included in the unconstrained generation dispatching, and not required to generate power by the power trading and dispatching center in the real-time operation, the price paid is $C_{p}$ only. $\mathrm{PSP}=\mathrm{PPP}+$ uplift. The uplift includes transmission losses, dispatching cost, ancillary service cost and costs incurred by transmission constraints due to non-merit based dispatching. In summary, the congestion management employed in the former England and Wales electricity market was done by redispatching generation units, and this resulted in the increase of the uplift and hence that of PSP.

\section{PROBLEM FORMULATION}

Suppose that the power system to be studied consists of $N$ independent GENCOs. To simplify the presentation, assume that each GENCO has only one registered unit (but the following method can be extended to general cases without technical difficulties). A GENCO builds a bid based on many factors such as the load forecasted, estimation of rivals' bidding behaviors, its own production cost and risk taking preference. Suppose GENCOs are required to bid a linear supply function and the bidding function of the $i$ th GENCO is $P_{i}=\alpha_{i}+\beta_{i} q_{i},\left(Q_{i \min } \leq q_{i} \leq Q_{i \max } ; i=1,2, \ldots, N\right)$. Here, $P_{i}$ $(\$ / \mathrm{MWh})$ is the bidding price at output $q_{i}, Q_{i \max }$ and $Q_{i \min }$ are the upper and lower limits of active power output respectively, $\alpha_{i}$ and $\beta_{i}$ are both bidding coefficients. The system load forecasted is $D$. After the power trading and dispatching center receives all the bids from GENCOs, the unconstrained generation dispatching is carried out first. Then, the generation dispatching is checked against the transmission capacity constraints. If transmission congestion occurs, the dispatching must be modified to remove the congestion, and this is the so-called congestion management procedure. The objective of the re-dispatching is to minimize the whole purchasing cost of energy. In this paper, only the problem of building the optimal bidding strategy for the oneperiod auction is addressed, and thus inter-temporal operating constraints for a generator, such as the minimum up and down times and the maximum number of start-ups and shutdowns allowed, are not accounted for.

Suppose that the $i$ th GENCO bids all its generation capacity $Q_{i \max }$, and there are several different scenarios of being dispatched as detailed below. If the dispatched output of the $i$ th GENCO in the unconstrained dispatching is $q_{i}\left(q_{i}=0\right.$ represents that the $i$ th GENCO is not dispatched) and this dispatching doesn't result in transmission congestion, the fee paid to this GENCO in a unit time is $\lambda q_{i}+C_{p} Q_{i \max }$. If the unconstrained dispatching results in transmission congestion, the dispatching must be modified and in this case suppose that the output increment of the ith GENCO, as a result of transmission congestion management, is $\Delta q_{i}$. When $\Delta q_{i}>0$, the fee paid to the GENCO in a unit time is $\lambda q_{i}+\int_{q_{i}}^{q_{i}+\Delta q_{i}}\left(\alpha_{i}+\beta_{i} x\right) d x+C_{p} Q_{i \max }$ and when $\Delta q_{i}<0$, the fee 
is $\lambda\left(q_{i}+\Delta q_{i}\right)+\int_{q_{i}+\Delta q_{i}}^{4_{i}}\left(\lambda-\alpha_{i}-\beta_{i} x\right) d x+C_{p} Q_{i \max }$.

If $U$ is used to denote the status of the transmission congestion $(U=0$ or 1 respectively indicates that the unconstrained dispatching does or does not result in transmission congestion), and $q_{i}+U \Delta q_{i}$ the actual dispatched output of the $i$ th GENCO, then a general formula representing the profit $\Omega$ of the $i$ th GENCO in a unit time, applicable to all scenarios described before, could be obtained as follow,

$$
\begin{aligned}
\Omega & =\lambda q_{i}+\int_{q_{i}}^{q_{i}+U \Delta q_{i}}\left(\alpha_{i}+\beta_{i} x\right) d x+C_{p} Q_{\text {max }}-C\left(q_{i}+U \Delta q_{i}\right) \\
& =\lambda q_{i}+\alpha U \Delta q_{i}+\frac{\beta_{i}}{2}\left[\left(q_{i}+U \Delta q_{i}\right)^{2}-q_{i}^{2}\right]+C_{p} Q_{\text {max }}-C\left(q_{i}+U \Delta q_{i}\right)
\end{aligned}
$$

where, $\mathrm{C}($.$) is the generation production cost function which is$ generally described as a quadratic one,

$$
C\left(q_{i}\right)=a_{i}+b_{1} q_{i}+c_{i} q_{i}^{2}
$$

$a_{i}, b_{i}$ and $c_{i}$ are generation production cost function coefficients.

Hence, for the $i$ th GENCO, the objective of building the optimal bidding strategy for a one-period auction is to determine optimal bidding coefficients $\alpha_{i}$ and $\beta_{i}$ to maximize its profit $\Omega$. The problem can be formulated as,

$$
\underset{\substack{\text { Mas. } \\ \text { Maximize }}}{ } \Omega
$$

Subject to:

$$
\left\{\begin{array}{l}
Q_{i \text { min }} \leq q_{i} \leq Q_{i_{\text {max }}} \\
\alpha_{i}+\beta_{i} q_{i} \leq \lambda_{\text {max }}
\end{array}\right.
$$

$\lambda_{\max }$ is the upper limit of the bidding price of GENCOs as specified by market trading protocols.

\section{Calculation of $\Omega$}

As stated before, after the power trading and dispatching center receives all bids from GENCOs, the unconstrained transmission dispatching is first carried out to determine the output level $q_{i}(i=1,2, \ldots, N)$ and the MCP $\lambda$. If the unconstrained dispatching results in transmission congestion, re-dispatching will be made to adjust the outputs of generators, i.e. to determine $\Delta q_{i}$.

\section{A. Calculations of $\lambda$ and $q_{i}$}

The unconstrained dispatching problem can be formulated as,

$$
\begin{gathered}
\alpha_{i}+\beta_{i} q_{i}=\lambda \quad(i=1,2, \cdots N) \\
\sum_{i=1}^{N} q_{i}=D
\end{gathered}
$$

For $q_{i}$, the inequality constraint as described in the first formula in Eqn. (4) must be respected. When the inequality constraints are ignored, $\lambda$ can easily be obtained by solving Eqns. (5) and (6),

$$
\lambda=\left(D+\sum_{i=1}^{N} \alpha_{i} / \beta_{i}\right) / \sum_{i=1}^{N} 1 / \beta_{i}
$$

The dispatched output of the $i$ th GENCO, $q_{i}$, can be obtained from Eqn. (5),

$$
q_{i}=\frac{1}{\beta_{i}} \lambda-\frac{\alpha_{i}}{\beta_{i}}
$$

When the solution of Eqn. (8) violates generation output limits, it must be modified to accommodate these limits. Specifically, when $q_{i}$ is smaller than its lower limit $Q_{i \min }, q_{i}$ should be set to zero rather than $Q_{\text {imin }}$ and the GENCO removed from the generation dispatching since the GENCO ceases to be competitive. When it is larger than the upper limit its value is set to $Q_{\text {inax }}$ and Eqn. (5) ignored for this GENCO since it is no longer a marginal generator. In these two cases, Eqn. (5) no longer holds for GENCO $i$. Obviously, $\lambda$ and $q_{i}$ are the functions of all GENCOs' bidding coefficients $\alpha_{i}$ and $\beta_{i}(i=I, 2, \ldots, N)$.

\section{B. Calculation of $\Delta q_{i}$}

As already stated before, if the unconstrained dispatching leads to transmission congestion, it must be modified to respect the transmission capacity constraints, i.e., to conduct the so-called congestion management to remove congestion, i.e. to determine the adjusted output $\Delta q_{i}(i=1,2, \ldots, N)$.

A congestion management model is presented here based on the congestion management mechanism introduced before. The objective is to minimize the uplift cost such incurred, i.e., the so-called congestion cost. Obviously, the congestion cost is the difference between the purchasing cost of the modified dispatching and that of the unconstrained dispatching.

The purchasing cost in the unconstrained dispatching is,

$$
\sum_{i=1}^{N}\left(\lambda \times q_{i}+C_{p} \times Q_{i \max }\right)
$$

The purchasing cost of the modified dispatching with the congestion management is,

$$
\begin{aligned}
& \sum_{i=1}^{N}\left(\lambda q_{i}+\int_{q_{i}}^{q_{i}+\Delta q_{i}}\left(\alpha_{i}+\beta_{i} x\right) d x+C_{p} Q_{i \max }\right) \\
& =\sum_{i=1}^{N}\left(\lambda q_{i}+\alpha_{i} \Delta q_{i}+\frac{\beta_{i}}{2}\left[\left(q_{i}+\Delta q_{i}\right)^{2}-q_{i}^{2}\right]+C_{p} Q_{i \max }\right)
\end{aligned}
$$

The congestion cost is the difference between Eqn. (10) and Eqn. (9),

$$
C_{c o n}=\sum_{i=1}^{N}\left(\alpha \cdot \Delta q_{i}+\frac{\beta_{i}}{2}\left[\left(q_{i}+\Delta q_{i}\right)^{2}-q_{i}^{2}\right]\right)
$$

Hence, the congestion management can be described as the following optimization problem,

$$
\underset{\Delta q_{2}, \Delta q_{2}, \cdots, \Delta q_{N}}{\operatorname{Minimize}} C_{\text {con }}
$$

Subject to:

1) Inequality constrains of generation outputs,

$$
Q_{i \min } \leq q_{i}+\Delta q_{i} \leq Q_{i_{\max }}
$$

2) Equality constraint of power balance,

$$
\sum_{i=1}^{N}\left(q_{i}+\Delta q_{i}\right)=D \text {, i.e. } \sum_{i=1}^{N} \Delta q_{i}=0
$$

3) Power flow constraints. Suppose that the DC power flow model is employed for the congestion management,

$$
\boldsymbol{\delta}=\mathbf{B}_{0}^{-1} \mathbf{P}
$$

$\boldsymbol{\delta}$ is the bus voltage phase angle vector, $\mathbf{B}_{\mathbf{0}}$ is the susceptance matrix and $\mathbf{P}$ net bus active power injection vector.

4) Transmission capacity constraints,

$$
P_{i j \text { new }} \leq P_{i j \max }
$$

$P_{i \text { nnew }}$ and $P_{i j \max }$ are the active power flow in branch $i-j$ 
after the congestion management is carried out and the upper limit of the transmission capacity of this branch.

The congestion management problem with Eqn. (12) as the objective function and Eqns. (13) through (16) as constraints is a quadratic planning one, and could easily be solved by existing methods.

\section{A MONTE CARLO SIMULATION BASED METHOD FOR BUILDING OPTIMAL BIDDING STRATEGIES}

As a preliminary study, suppose that, from the $i$ th GENCO's point of view, the bidding coefficients of the $j$ th rival, $\alpha_{j}$ and $\beta_{j}(j=1,2, \ldots, N ; j \neq i)$, obey a joint normal distribution with the following probability density function (pdf $^{[3]}$,

$$
\begin{aligned}
& p d f_{i}\left(\alpha_{j}, \beta_{j}\right)=\frac{1}{2 \pi \sigma_{j}^{(\alpha)} \sigma_{j}^{(\beta)} \sqrt{I-\rho_{j}^{2}}} \times \\
& \left.\exp \left\{\begin{array}{l}
-\frac{1}{2\left(1-\rho_{j}^{2}\right)}\left[\left(\frac{\alpha_{j}-\mu_{j}^{(\alpha)}}{\sigma_{j}^{(\alpha)}}\right)^{2}-\frac{2 \rho_{j}\left(\alpha_{j}-\mu_{j}^{(\alpha)}\right)\left(\beta_{j}-\mu_{j}^{(\beta)}\right)}{\sigma_{j}^{(\alpha)} \sigma_{j}^{(\beta)}}\right] \\
+\left(\frac{\beta_{j}-\mu_{j}^{(\beta)}}{\sigma_{j}^{(\beta)}}\right)^{2}
\end{array}\right]\right\}
\end{aligned}
$$

The methods that could be used to determine these probability density functions are discussed briefly in Ref. [3] and will not be repeated here. The meanings of the symbols in Eqns. (17) are exactly the same as those in Ref. [3].

By using the probability density function to describe the rivals' bidding behaviors, the problem of building the optimal bidding strategy with Eqn. (3) as the objective function and Eqn. (4) as constraints then becomes a stochastic optimization problem, and could be solved by the well-known Monte Carlo simulation method. The Monte Carlo simulation method is generally used to provide numerically approximate solutions to mathematical, physical and engineering problems by performing stochastic simulations and statistical sampling experiments on a computer. The basic procedure of solving a stochastic optimization problem using this method is as follows. First, generate plenty of random samples according to the distributions of random variables in the problem studied on a computer. Secondly, obtain plenty of trial outcomes by solving the problem with all random variables taking sampling values. Finally, calculate the statistic parameters such as the expectation value or standard deviation of the trial outcomes. Usually the expectation value is taken as the final solution to the problem.

Hence, the procedure of building the optimal bidding strategy for the $i$ th GENCO, i.e. determining the optimal bidding coefficients, by using the Monte Carlo method, is as follows. First, generate many random samples according to the distributions of random variables, $\alpha_{j}$ and $\beta_{j}(j=1,2, \ldots, N$; $j \neq i$ ). For each Monte Carlo simulation iteration, the optimization problem described as (3) through (4) becomes a definite optimization problem, and the well-known Genetic Algorithm (GA) is employed for solving this problem. Thus, in each simulation, a set of optimal, $\alpha_{j}$ and $\beta_{j}$ could be found. After a pre-specified number of simulations is carried out, the expectation values of $\alpha_{j}$ and $\beta_{j}$ are taken as the optimal bidding coefficients, i.e. the optimal bidding strategy.

\section{NUMERICAL EXAMPLES}

Two numerical examples are used to illustrate the proposed method.

\section{A. Example I}

A very simple two-bus system with two generation units (GENCOs) included, as shown in Fig.1, is first employed. The production cost functions of the two GENCOs, i.e. Gl and $\mathrm{G} 2$, are $C_{1}\left(q_{1}\right)=4.68 q_{1}+0.0312 q_{1}^{2}$ and $C_{2}\left(q_{2}\right)=9.36 q_{2}+$ $0.1092 q_{2}{ }^{2}$, respectively. The upper and lower limits of generation outputs of these two generation units are both 200 MW and $30 \mathrm{MW}$, respectively. There are two loads, i.e. $\mathrm{L}_{1}=40 \mathrm{MW}$ and $\mathrm{L}_{2}=150 \mathrm{MW}$, and they are both inelastic to the price of electricity. LOLP is set to be 0.001 , and VLL $2 £ k W h$, or approximately $1282 \$ / \mathrm{MWh}$. The upper limit of the bidding price by GENCOs, i.e. $\lambda_{\max }$, is $250 \$ / \mathrm{MWh}$..

The rival GENCO is assumed to have an estimated joint normal distribution for the two bidding coefficients. For the $i$ th GENCO $(i=1,2)$, the estimated parameters in the joint normal distributions for the $j$ th rival GENCO $(j=1,2$ and $j \neq i)$, as described in Eqn. (17), are specified in the Monte Carlo simulations as (18).

$$
\begin{aligned}
& \mu_{j}^{(\alpha)}=1.2 \mathrm{~b}_{\mathrm{j}}, \quad 4 \sigma_{j}^{(\alpha)}=0.15 \mathrm{~b}_{\mathrm{j}} \Rightarrow \sigma_{j}^{(\alpha)}=0.15 \mathrm{~b}_{\mathrm{j}} / 4 ; \\
& \mu_{j}^{(\beta)}=1.2 \times 2 \mathrm{c}_{\mathrm{j}}, 4 \sigma_{j}^{(\beta)}=0.15 \mathrm{c}_{\mathrm{j}} \Rightarrow \sigma_{j}^{(\beta)}=0.15 \mathrm{c}_{\mathrm{j}} / 4, \rho_{j}=-0.1
\end{aligned}
$$

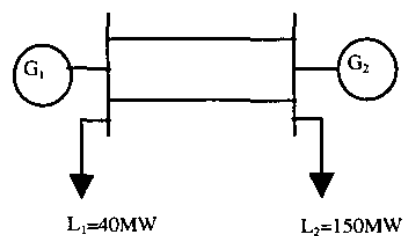

Fig.1 A 2-bus system

It should be mentioned that the parameters in Eqn. (18) ould be estimated using available mathematical methods (such as statistical methods) based on sufficient historical bidding data. These parameters are specified here to illustrate the basic feature of the method developed. Detailed explanations about these specifications are given in Ref. [3].

Many simulation tests were carried out using the Monte Carlo method, and the number of simulations is specified to be 10000 . For each simulation, the bidding coefficients of rivals are fixed and the optimal bidding coefficients of the studied GENCO are then determined using the GA. The realvalued encoding method is utilized in the GA implementation. The parameters associated with the GA are specified as follows: the population size is 20 , and the maximum permitted number of iteration is 30 . A set of the optimal bidding coefficients $a_{j}$ and $\beta_{j}$ can then be found in each simulation. Finally, their expectation values are used as the optimal bidding coefficients.

Two cases are tested with different transmission capacities to illustrate the proposed method and the impacts of 
transmission constraints on the optimal bidding strategies of GENCOs.

\section{1) Case 1}

The transmission capacities of the two lines are both 100 MW. Obviously, transmission congestion will not happen no matter how the two GENCOs bid. The optimal bidding strategies of the two GENCOs obtained by the proposed method are:

$$
\alpha_{1}=21.8641, \beta_{1}=0.1410 ; \alpha_{2}=21.1615, \beta_{2}=0.2704
$$

If the two GENCOs bid according to these optimal bidding strategies, the generation output dispatched are $q_{l}=123.2 \mathrm{MW}$ and $q_{2}=66.8 \mathrm{MW}$, respectively, and the MCP is $39.23 \$ / \mathrm{MWh}$ The profits of two GENCOs are $\Omega_{1}=4030.6 \$$ and $\Omega$ ${ }_{2}=1756.7 \$$. The power flow in each transmission line is 41.6 MW. In this case, there is no need to activate the congestion management program.

2) Case 2

Now, let us reduce the transmission capacity of each line to $50 \mathrm{MW}$. In this case, the obtained optimal bidding strategies of the two GENCOs are,

$$
\alpha_{1}=21.8542, \beta_{1}=0.1411 ; \alpha_{2}=90.0645, \beta_{2}=0.7990
$$

Compared with the results in Case 1 , the optimal bidding strategy of $G_{1}$ is similar, while the bidding price of $G_{2}$ is enhanced obviously.

If the two GENCOs bid according to these optimal strategies, the dispatched outputs in the unconstrained dispatching are $q_{l}=190 \mathrm{MW}$ and $q_{2}=0 \mathrm{MW}$, and the MCP is $48.66 \mathrm{\$} / \mathrm{MWh} . \mathrm{G}_{2}$ is not dispatched because of its high bidding price. However, in this case the total power flow of these two lines is $150 \mathrm{MW}$, and this exceeds the upper limit of the total transmission capacity, i.e. 100MW. In other word, transmission congestion occurs. Thus, $\mathrm{G}_{1}$ can only supply 100 $\mathrm{MW}$ to $\mathrm{L}_{2}$, and the left $50 \mathrm{MW}$ of $\mathrm{L}_{2}$ must be provided by $\mathrm{G}_{2}$. The adjusted outputs of the two GENCOs by the congestion management program are $\triangle q_{1}=-50 \mathrm{MW}$ and $\triangle q_{2}=50 \mathrm{MW}$. Since the $50 \mathrm{MW}$ supplied by $\mathrm{G}_{2}$ is paid at its own bidding price, the higher price it bids, the larger profit it can obtain (note that the bidding price cannot exceed the upper limit of the bidding price, as defined by the market rules). Hence, the actually dispatched outputs of the two GENCOs are $q_{1}=50$ MW and $q_{2}=0 \mathrm{MW}$, and the profits of them are $\Omega_{1}=5968.4 \$$ and $\Omega_{2}=5007.6 \$$, respectively.

Obviously, when the transmission capacity is insufficient, a local submarket could actually be formed at the L2 side. As a result, G2 will be a monopoly in this submarket, and could raise its bidding price to a very high degree, subject to the price cap limitation. It is shown by this very simple example that transmission congestion could divide the whole market into two or more submarkets and hence remarkably change the competitive positions of GENCOs in the electricity market.

\section{B. Example II}

This example is the IEEE 14-bus system. The system parameters are given in the Ref. [11]. ne generation unit. Parameters of the five generation units are listed in Table I. For the $i$ th GENCO $(i=1,2,3,4,5)$, assume that the bidding parameters of its $j$ th rival $(j=1,2,3,4,5$ and $j \neq i)$ obey joint normal distribution and the expectation values and standard deviations are also specified as in Eqn. (18) The number of the Monte Carlo simulations is specified to be 10000 and the parameters of the GA are the same as those in Example I. The optimal bidding strategies of the five GENCOs are listed in Table II.

\begin{tabular}{cccccccc}
\multicolumn{7}{c}{ TABLE I UNIT PARAMETERS OF GENERATION COMPANIES } \\
\hline GENCO & $a_{i}$ & $b_{i}$ & $c_{i}$ & $\begin{array}{c}\text { Upper } \\
\text { limit of } \\
\text { unit } \\
\text { output }\end{array}$ & $\begin{array}{c}\text { Lower } \\
\text { limit of } \\
\text { unit } \\
\text { output }\end{array}$ & $\begin{array}{c}\text { node } \\
\text { No. }\end{array}$ \\
\hline $\mathrm{I}$ & 0 & 3.0 & 0.1375 & 15 & 90 & 1 \\
2 & 0 & 5.25 & 0.0525 & 20 & 100 & 2 \\
3 & 0 & 3.0 & 0.0875 & 20 & 100 & 3 \\
4 & 0 & 5.0 & 0.075 & 20 & 120 & 6 \\
5 & 0 & 4.0 & 0.075 & 15 & 100 & 8 \\
\hline
\end{tabular}

If all GENCOs bid according to these optimal bidding strategies showed in Table 2, the dispatched outputs (in MW) of the five generation units by the unconstrained dispatching are $q_{1}=44.4, q_{2}=73.7, q_{3}=63.8, q_{4}=61.0, q_{5}=6.1$.

The MCP is $16.94 \$ / \mathrm{MWh}$.

TABLE II OPTIMAL BIDDING STRATEGIES OF GENERATION COMPANIES

\begin{tabular}{ccc}
\hline GENCO & \multicolumn{2}{c}{ optimal bidding strategy } \\
\hline 1 & 6.4002 & $\beta_{j}$ \\
2 & 8.7998 & 0.2374 \\
3 & 6.6424 & 0.1104 \\
4 & 8.1817 & 0.1615 \\
5 & 7.5056 & 0.1436 \\
\hline
\end{tabular}

The unconstrained dispatching is then checked against transmission capacities by applying the DC power flow computation, and it is found that no congestion occurs. Hence this dispatch is technically feasible. The profits (\$) of the five GENCOs are,

$\Omega_{1}=461.8, \Omega_{2}=703.1, \Omega_{3}=659.7, \Omega_{4}=601.1, \Omega_{5}=654.3$

Now, let us see how GENCOs will bid if transmission congestion is expected to happen. For this purpose, the transmission capacities of branch 3 and 17 are supposed to decrease from $1.71^{[11]}$ to 0.25 (in p.u.). Other system parameters remain unchanged. The optimal bidding strategies for the five GENCOs obtained by the proposed method are showed in Table III. If all the five GENCOs bid according to the optimal bidding strategies listed in Table 3, the dispatched outputs (in $\mathrm{MW}$ ) of the five generation units by the unconstrained dispatching are,

$$
q_{1}=42.2, q_{2}=100.0, q_{3}=66.8, q_{4}=0, q_{5}=100.0
$$

TABLE III OPTIMAL BIDDING STRATEGIES OF GENCOS UNDER TRAANSIMISSION CONGESTION

\begin{tabular}{ccc}
\hline & \multicolumn{2}{c}{ optimal bidding strategy } \\
GENCO & $\alpha_{j}$ & $\beta_{j}$ \\
\hline 1 & 13.8263 & 0.6552 \\
2 & 16.6098 & 0.0795 \\
3 & 13.9608 & 0.4121 \\
4 & 23.5718 & 0.3510 \\
5 & 4.9148 & 0.1051 \\
\hline
\end{tabular}

The MCP is $41.48 \$ / \mathrm{MWh}$. GENCO 4 is not dispatched due to its high bidding price and the bidding capacity of GENCO 5 is fully dispatched because of its lowest bidding price among all GENCOs. However, when this dispatch is checked against transmission capacities by the DC power flow computation, it is found that the power flow in branch 3 is 0.3292 and that in branch 170.4833 and the transmission 
constraints in both branches are violated, or in other word, congestions occur in these two branches. The adjusted outputs of the five GENCOs by the congestion management program are $\triangle q_{1}=27.3, \triangle q_{2}=-60.9, \triangle q_{3}=-1.4, \triangle q_{4}=120.0, \triangle q_{5}=-85.0$.

The bidding capacity of GENCO 4 is fully dispatched, while the output of GENCO 5 is remarkably cut down. Transmission congestions in branch 3 and branch 17 are removed in the modified generation dispatch, and the current power flows in these two branches are both 0.25 . Finally, the dispatched outputs of the five GENCOs are,

$$
q_{1}=69.5, q_{2}=39.1, q_{3}=65.4, q_{4}=120, q_{\mathrm{s}}=15.0
$$

The profits of these GENCOs are, $\Omega_{1}=2367.5, \Omega_{2}=2637.9, \Omega_{3}=2267.4, \Omega_{4}=3824.4, \Omega_{5}=3264.1$

From these results, it is obvious that the transmission congestions lead to a remarkable change of the competitive position of GENCO 4 in the electricity market, and have great impacts on the bidding strategies of generation companies.

\section{CONCLUDING REMARKS}

The optimal bidding strategies for generation companies in the competitive electricity market environment are investigated in this paper with transmission capacity constraints taken into account. The problem is formulated as a stochastic optimization model and solved by the Monte Carlo simulation method and genetic algorithm. It should be mentioned that the emphasis of this paper is on developing a methodological framework for solving this problem, rather than on how to estimate the bidding behaviors of rivals. Two examples are employed to illustrate the proposed method, and it is showed that generation companies could utilize transmission congestions to manipulate the market. The proposed method could also be served for roughly analyzing the potential of market power abusing by generation companies under the circumstances of transmission congestions.

\section{REFERENCES}

11] A. K. David and F. S. Wen, "Strategic Bidding in Competitive Electricity Markets: a Literature Survey," in Proc. 2000 IEEE PES Summer Power Meeting, vol.4, pp.2168-2173.

[2] A. K. David, "Competitive Bidding in Electricity Supply", IEE Proceedings-Generation Transmission \& Distribution, vol. 140. No. 5.pp. 421-426. Sep. 1993.

[3] F. S. Wen, A. K. David. "Optimal Bidding Strategies and Modeling of Imperfect Information Among Competitive Generators", IEEE Trans on Power Systems, vol. 16, No. 1, pp. 15-21, Feb. 2001

[4] F. S. Wen. A. K. David, "Coordination of Bidding Strategies in Day-ahead Energy and Spinning Reserve Markets", International Journal of Electrical Power and Energy System, vol. 24, No. 4, pp 251-261, 2002.

[5] H. L. Song, C. C. Liu, J. Lawarree, "Optimal Electricity Supply Bidding by Markov Decision Process", IEEE Trans on Power Systems, vol.15, No. 2, pp. 618-624, May 2000

[6] S. Hao, "A Study of Basic Bidding Strategy in Clearing Pricing Auctions", IEEE Trans on Power Systems, vol. 15, No 3, pp. 975-980, Aug. 2000.

[7] H. Singh, S. Hao, A. Papalexopoulos, "Power Auctions and Network Constraints", in: Proceedings of the Thirtieth Hwaii Intemational Conference on System Sciences. Hwaii (USA), pp. 608-614, 1997.

[8] G. A. Berry, B. F. Hobbs, W. A. Meroney, et al., "Analyzing Strategic Bidding Behavior", in Transmission Networks", In: IEEE Tutorial on Game Theory Applications in Electric Power Markets (Editor: Singh H), IEEE Power Engineering Society, pp.7-32, 1999.

[9] K. L. Lo, Y. S. Yuen, "Congestion Management in Deregulated Electricity Markets", In: Proceedings of International Conference on Electric Utility
Deregulation and Restructuring and Power Technologies". (DRPT 2000), London (UK), pp. 47-52, 2000.

[10] A. K. David and F. S. Wen, "Market Power in Electricity Supply", IEEE Trans on Energy Conversion, vol. 16, No. 4, pp. 352-360, Dec. 2001.

[11] B. M. Zhang, S. S. Chen, "Power Grid Analysis". Tsinghua University Press, May 1996

\section{BiographiES}

Li Ma received her BE and ME degrees in electrical engineering from Shandong University, China, in 1996 and 2000, respectively. Since 2000, she has been working for her $\mathrm{Ph} \mathrm{D}$ in electrical engineering in Zhejiang University, China Her main area of interest is power industry restructuring.

Fushuan Wen received his $\mathrm{BE}$ and $\mathrm{ME}$ degrees from Tianjin University, China, in 1985 and 1988, respectively, and $\mathrm{PhD}$ from Zhejiang University, China, in 1991, all in electrical engineering. He joined the faculty of Zhejiang Unversity, China, in 1991, and has been professor there since 1997. He held a visiting position in the National University of Singapore from 1995 to 1997. and was a research fellow in the Hong Kong Polytechnic University from July 1998 to February 2001 . He is now a research assistant professor in the University of Hong Kong. His current research interests are in power industry restructuring and artificial intelligence applications in power systems.

Yixin Ni (Senior Member. IEEE) received her B. Eng., M. Eng., and Dr. Eng. all from Electrical Engineering Department, Tsinghua University, P.R. China in 1968, 1981 and 1983 respectively. Her research interests are in power system modeling, simulation, stability and control, and power electronics applications in power systems. She was a professor of Tsinghua University and is now with the University of Hong Kong. She is a senior member of CSEE and Fellow of HKIE

Felix F. Wu (Fellow, IEEE) received his $\mathrm{Ph}$. D. from University of California at Berkeley (UCB). He is now a chair professor of electrical engineering at the University of Hong Kong. Prior to that he was a professor and vice chairman of Dept. of EECS. UCB. His research interests are in power industry restructuring, power system investment planning, design of modem control centers, distribution automation, distributed processing etc. 